\title{
Comparación de la evaluación clínica y por ultrasonido de la artropatía hemofílica en pacientes pediátricos
}

Guadalupe Ortiz*, Edith Andrade, Álvaro Vargas, Bernardo Ramírez, Berenice Sánchez y

Octavio Martínez

Servicio de Hematología, Hospital General "Dr. Gaudencio González Garza", Centro Médico Nacional La Raza, Instituto Mexicano del Seguro Social, Ciudad de México, México

\section{Resumen}

Antecedentes: La exploración articular por ultrasonido mediante el método HEAD-US en la detección de la artropatía temprana ha sido poco estudiada en nuestro país. Objetivo: Comparar la evaluación clínica y por ultrasonido de las articulaciones en niños con hemofilia. Métodos: Estudio longitudinal, prospectivo y descriptivo con pacientes pediátricos con hemofilia $A$ y $B$ valorados con la escala HJHS 2.1 y ultrasonido con transductor lineal de 8 a $12 \mathrm{MHz}$. Se evaluaron las articulaciones de codos, rodillas y tobillos de forma bilateral, con el método HEAD-US. Resultados: Se incluyeron 69 pacientes; de ellos, 48 con hemofilia A grave (peso: $40.1 \mathrm{~kg}$ ). En la escala HJHS se observó mayor afectación en la rodilla izquierda (0.49) y menor en el tobillo derecho (0.05). Con la escala HEAD-US, la más afectada fue la rodilla derecha (0.78). Existe una relación significativa en la afectación de la rodilla derecha evaluada con la escala HEAD-US en presencia de inhibidor. Conclusiones: EI peso superior al percentil 50 es un factor de riesgo independiente de complicaciones por sangrado articular, mientras que la edad y el tipo de hemofilia no parecen relacionados. El método HEAD-US es una herramienta útil y accesible para la detección temprana de artropatía y hemartrosis.

PALABRAS CLAVE: Hemofilia. Artropatía. HEAD-US. Ultrasonido. Articulación.

\section{Comparison of the clinical and ultrasound evaluation of haemophilic arthropathy in children}

\begin{abstract}
Background: Joint ultrasound examination using the HEAD-US method in the detection of early arthropathy is poorly studied in our country. Objective: To compare the clinical and ultrasound evaluation of the joints in haemophilia. Methods: Longitudinal, prospective and descriptive study with paediatric patients with haemophilia A and B evaluated with the HJHS 2.1 scale and ultrasound with a linear transducer of 8 to $12 \mathrm{MHz}$. Elbows, knees and ankles joints were evaluated bilaterally, with $H E A D$ US protocol. Results: 69 paediatric patients were included of which 48 with severe haemophilia A (weight: $40.1 \mathrm{~kg}$ ). On the HJHS scale, a greater involvement was observed in the left knee (0.49), and less in the right ankle (0.05). With the HEAD-US scale, the most affected was the right knee (0.78). There is a significant relationship in the involvement of the right knee evaluated with the HEAD-US scale in the presence of inhibitor. Conclusions: Weight above the $50^{\text {th }}$ percentile is an independent risk factor for joint bleeding complications, while age and type of haemophilia do not appear to be related. The HEAD-US method is a useful and accessible tool for early detection of arthropathy and hemarthrosis.
\end{abstract}

KEYWORDS: Haemophilia. Arthropathy. HEAD-US. Ultrasound. Joint.

Correspondencia:

*Guadalupe Ortiz

E-mail: dcortizt@yahoo.com.mx
Gac Med Mex. 2022;158:12-16

Disponible en PubMed www.gacetamedicademexico.com

0016-3813/@ 2021 Academia Nacional de Medicina de México, A.C. Publicado por Permanyer. Este es un artículo open access bajo la licencia CC BY-NC-ND (http://creativecommons.org/licenses/by-nc-nd/4.0/). 


\section{Introducción}

La hemartrosis es la manifestación hemorrágica más frecuente y de mayor morbilidad del paciente hemofílico, con una prevalencia del $80 \%$. Se presenta con sinovitis y daño articular progresivo e irreversible, ocasionando dolor crónico, incapacidad funcional y discapacidad permanente. ${ }^{1,2}$ Las articulaciones mayormente afectadas son las de rodillas, codos y tobillos. ${ }^{1}$ Se manifiesta por dolor, rigidez, rubor y distensión articular, con limitación y contractura muscular hasta la flexión permanente. ${ }^{3}$

La valoración de la hemartrosis mediante imagen puede realizarse por distintos métodos. La radiografía muestra erosión ósea, quistes subcondrales y disminución del espacio articular. La resonancia magnética aporta excelente resolución tisular, puede identificar el origen del sangrado y detecta alteraciones precoces en pacientes con pocos episodios de sangrado; sin embargo, es un método con un alto costo, poca disponibilidad y en pacientes pequeños requiere sedación. ${ }^{4}$ La exploración articular por ultrasonido mediante el método HEAD-US (Haemophilia Early Arthropathy Detection with Ultrasound) ${ }^{5}$ es de gran valor en los pacientes con hemofilia, por ser una herramienta extensamente disponible, de fácil manejo, no invasiva, que no requiere sedación, de bajo costo y efectiva para la identificación de sinovitis crónica y aguda, daño subcondral y artropatía crónica. También es de utilidad en el seguimiento y para realizar ajustes de tratamiento, y una excelente guía en los procedimientos de artrocentesis. ${ }^{6,7}$

Presentamos los resultados de un estudio realizado en la clínica de hemofilia del Centro Médico Nacional La Raza para comparar los resultados de la evaluación clínica con los de la evaluación por ultrasonografía con el método HEAD-US en pacientes pediátricos con hemofilia de los tipos A y $B$.

\section{Métodos}

Estudio longitudinal, prospectivo, analítico y descriptivo. Se incluyeron pacientes varones con edades comprendidas entre 1 y 16 años, con diagnóstico de hemofilia tipo A o B clasificada como grave, moderada o leve. Se realizó un análisis descriptivo para comparar las características clínicas y ultrasonográficas de las articulaciones de codos, rodillas y tobillos. El estudio se realizó en el Servicio de
Hematología Pediátrica del Centro Médico Nacional La Raza, en el periodo comprendido del 1 de agosto de 2018 al 1 de diciembre de 2018. El protocolo del estudio fue aprobado por el Comité Local de Investigación en Salud del Instituto Mexicano del Seguro Social (IMSS) con número de registro R-2019-3502-098. Se respetaron los principios éticos según los estándares internacionales para investigación en seres humanos (Declaración de Helsinki de la Asociación Médica Mundial, Constitución Política de los Estados Unidos Mexicanos y artículo 17 de la Ley General de Salud en Materia de Investigación para la Salud).

\section{Exploración articular}

Se realizó a cada paciente una evaluación clínica con la escala HJHS 2.1 (Hemophilia Joint Health Score) por médicos hematólogos pediatras y a la par se realizó valoración articular por ultrasonido con transductor lineal de 8 a $12 \mathrm{MHz}$, modelo $\mathrm{R} 7$ de la marca Samsung, por médicos radiólogos y hematólogos pediatras previamente adiestrados. Se evaluaron las articulaciones de codos, rodillas y tobillos de forma bilateral, siguiendo el método HEAD-US. ${ }^{5}$

\section{Análisis estadístico}

Las variables cualitativas binomiales se analizaron con prueba de ji al cuadrado o prueba exacta de Fisher, y las cuantitativas sin distribución normal se analizaron con la prueba $U$ de Mann-Whitney y la prueba de Kruskal-Wallis. Se utilizaron los programas Excel y SPSS versión 20.

\section{Resultados}

Se incluyeron en el estudio 69 pacientes con una edad media de 10 años; el tipo de hemofilia más frecuente fue el tipo A grave. Los pacientes fueron distribuidos por edades en tres grupos: 1-5 años, 6-10 años y 11-15 años. La media de peso fue de 16.4 para el grupo de 1-5 años, $25.3 \mathrm{~kg}$ para el grupo de 6-10 años y $40.6 \mathrm{~kg}$ para el grupo de 11-15 años. En el momento de la evaluación, 40 pacientes se encontraban bajo profilaxis secundaria y solo 5 estaban en tratamiento oportuno a demanda. Al evaluar la presencia de inhibidor se constató positividad en 7 (14\%) pacientes con hemofilia tipo A grave y $2(13 \%)$ con hemofilia tipo $B$ grave (Tabla 1). 
Tabla 1. Características demográficas de la población estudiada

\begin{tabular}{|c|c|c|c|c|}
\hline Edad & $1-5$ años & 6-10 años & \multicolumn{2}{|c|}{ 11-15 años } \\
\hline Peso (media) & \multicolumn{4}{|c|}{$9.8-23 \mathrm{~kg}(16.4) 23-57$ kg (23.5) 36-88 kg (40.6) } \\
\hline \multirow{4}{*}{$\begin{array}{l}\text { Tipo de } \\
\text { hemofilia y } \\
\text { gravedad }\end{array}$} & & & A & B \\
\hline & \multicolumn{2}{|l|}{ Leve } & 4 & 1 \\
\hline & \multicolumn{2}{|l|}{ Moderada } & 2 & 3 \\
\hline & \multicolumn{2}{|l|}{ Grave } & 48 & 11 \\
\hline \multirow[t]{2}{*}{$\begin{array}{l}\text { Tipo de } \\
\text { tratamiento }\end{array}$} & $\begin{array}{l}\text { Profilaxis } \\
\text { primaria }\end{array}$ & $\begin{array}{l}\text { Profilaxis } \\
\text { secundaria }\end{array}$ & $\begin{array}{c}\text { Profilaxis } \\
\text { terciaria }\end{array}$ & $\begin{array}{l}\text { Oportuno a } \\
\text { demanda }\end{array}$ \\
\hline & 23 & 40 & 1 & 5 \\
\hline \multirow{2}{*}{$\begin{array}{l}\text { Presencia de } \\
\text { inhibidor y tipo } \\
\text { de hemofilia }\end{array}$} & A & \multicolumn{3}{|c|}{ B } \\
\hline & 7 & \multicolumn{3}{|c|}{2} \\
\hline
\end{tabular}

\section{Evaluación clínica de acuerdo con la escala HJHS 2.1}

La articulación con mayor afectación por las complicaciones de la enfermedad fue la rodilla izquierda, con un puntaje promedio obtenido en la muestra de 69 niños de 0.49 (intervalo de confianza del 95\% [IC 95\%]: 0-11), seguida por la rodilla derecha, el codo izquierdo y el codo derecho. La articulación menos comprometida fue el tobillo derecho, con un puntaje promedio de 0.05 (IC 95\%: 0-2).

\section{Evaluación por ultrasonido de acuerdo con la escala HEAD-US}

La articulación más afectada fue la rodilla derecha, con un puntaje medio de 0.78 (IC 95\%: 0-5), seguida por la rodilla izquierda y el tobillo derecho.

Al comparar la correlación de la escala clínica cuando existe daño articular con la escala por ultrasonido se observó una elevada probabilidad de presencia de artropatía (Tabla 2).

Al evaluar la artropatía en la hemofilia tipo A grave se obtuvieron los puntajes más bajos en ambas escalas. Únicamente se observó una relación estadísticamente significativa en la afección de la rodilla derecha $(p=0.017)$ y del tobillo izquierdo $(p=0.002)$ cuando se valoró con la escala clínica (Tabla 3).

Tomando en consideración la presencia de inhibidor, al evaluar la artropatía por ambas escalas se observó una relación estadísticamente significativa $(p=0.019)$ en la afectación de la rodilla derecha al evaluar con la escala HEAD-US (Tabla 4).
Tabla 2. Evaluación de acuerdo con la escala clínica y por ultrasonido

\begin{tabular}{|c|c|c|c|}
\hline Articulaciones & $\begin{array}{c}\text { Clínica HJHS } \\
2.1\end{array}$ & HEAD-US & $\begin{array}{c}p(\text { de de } \\
\text { Mann-Whitney) }\end{array}$ \\
\hline $\begin{array}{c}\text { Derechas } \\
\text { Codo }\end{array}$ & $0.3043( \pm 0.9)$ & $0.4058( \pm 1.2)$ & 0.0002 \\
$\begin{array}{l}\text { Rodilla } \\
\text { Tobillo }\end{array}$ & $0.4493( \pm 1.2)$ & $0.7826( \pm 1.7)$ & 0.0007 \\
\hline $\begin{array}{c}\text { Izquierdas } \\
\text { Codo }\end{array}$ & $0.0580( \pm 0.2)$ & $0.2899( \pm 0.8)$ & 0.0001 \\
\hline $\begin{array}{c}\text { Rodilla } \\
\text { Tobillo }\end{array}$ & $0.3333( \pm 0.9)$ & $0.5072( \pm 1.2)$ & 0.0002 \\
\hline
\end{tabular}

HEAD-US: Hemophilia Early Arthropathy Detection with Ultrasound; HJHS: Hemophilia Joint Health Score.

Tabla 3. Hemofilia tipo A grave y relación entre las dos escalas

\begin{tabular}{|l|c|c|}
\hline Sitio afectado & HJHS 2.1 & HEAD-US \\
\hline Codo derecho & $p$ & $p$ \\
\hline Codo izquierdo & 0.997 & 1.00 \\
\hline Rodilla derecha & 0.949 & 1.00 \\
\hline Rodilla izquierda & 0.017 & 0.177 \\
\hline Tobillo derecho & 0.966 & 0.979 \\
\hline Tobillo izquierdo & 0.999 & 0.999 \\
\hline
\end{tabular}

HEAD-US: Hemophilia Early Arthropathy Detection with Ultrasound; HJHS: Hemophilia Joint Health Score.

Al valorar la artropatía con ambas escalas en función de la edad se observó que el grupo de 6-10 años fue el que presentó puntajes más bajos, seguido del grupo de 11-15 años. La rodilla izquierda fue la que mostró mayor afección $(p=0.007)$ al evaluarla con la escala clínica (Tabla 5).

\section{Discusión}

El presente trabajo de investigación fue diseñado para definir si el ultrasonido articular con método de exploración HEAD-US en pacientes con hemofilia A y $B$ es una herramienta útil para detectar la artropatía temprana cuando la exploración con la escala clínica HJHS 2.1 es negativa, y de esa forma prevenir la evolución de la enfermedad a etapas avanzadas.

En nuestro estudio se utilizó el método HEAD-US, diseñado como herramienta rápida accesible para evaluación articular, por los antecedentes de estudios 
Tabla 4. Presencia de inhibidor y relación entre las dos escalas

\begin{tabular}{l|c|c|}
\hline Sitio afectado & HJS & HEAD-US \\
\cline { 2 - 3 } & $p$ & $p$ \\
\hline Codo derecho & 0.673 & 0.896 \\
\hline Codo izquierdo & 0.687 & 0.110 \\
\hline Rodilla derecha & 0.765 & 0.019 \\
\hline Rodilla izquierda & 0.314 & 0.329 \\
\hline Tobillo derecho & 0.838 & 0.802 \\
\hline $\begin{array}{l}\text { Tobillo izquierdo } \\
\text { HEAD-US: Hemophilia Early Arthropathy Detection with Ultrasound; HJHS: Hemophilia } \\
\text { Joint Health Score. }\end{array}$ & & 0.840 \\
\hline
\end{tabular}

Tabla 5. Relación entre las escalas HJHS 2.1 y HEAD-US

\begin{tabular}{|l|c|c|}
\hline Sitio afectado & HJHS 2.1 & HEAD-US \\
\cline { 2 - 3 } & $\mathbf{p}$ & $\mathbf{p}$ \\
\hline Codo derecho & 0.578 & 0.556 \\
\hline Codo izquierdo & 0.232 & 0.272 \\
\hline Rodilla derecha & 0.508 & 0.227 \\
\hline Rodilla izquierda & 0.007 & 0.519 \\
\hline Tobillo derecho & 0.504 & 0.154 \\
\hline Tobillo izquierdo & 0.639 & 0.371 \\
\hline
\end{tabular}

HEAD-US: Hemophilia Early Arthropathy Detection with Ultrasound; HJHS: Hemophilia Joint Health Score.

previos como el publicado por De la Corte-Rodríguez, et al. ${ }^{1,2}$ en el que se observó un amplio porcentaje de pacientes sin historia de sangrado articular documentada que en la escala HJHS 2.1 presentaban una puntuación de $0, y$ al practicarles una exploración ultrasonográfica con el método HEAD-US presentaron cambios tempranos de daño articular y hemartrosis, indicando la idoneidad de este método para la detección precoz de signos de daño articular. Nuestros resultados están en concordancia con sus hallazgos.

Respecto a la afectación articular, los resultados de nuestro estudio indican que las rodillas son la articulación más afectada y con manifestaciones más graves. Además, se observó que existe una estrecha correlación entre los sangrados articulares recurrentes y las complicaciones de hemartrosis en los pacientes sin profilaxis primaria con inhibidor, mientras que los pacientes que recibían profilaxis fueron los que mostraron menor correlación. De manera adicional, observamos que en nuestra población con hemofilia hay tendencia al sobrepeso (por arriba del percentil 50). Esto implica que en la edad pediátrica aún no logramos incidir en los adecuados cambios de alimentación y estilo de vida, lo que a su vez repercutirá en una mayor incidencia de artropatía en la etapa de adulto.

De acuerdo con las estadísticas europeas y americanas, se ha observado una incidencia de desarrollo de inhibidor del $25-40 \%$ para la hemofilia $A$ y del $1-5 \%$ para la hemofilia $\mathrm{B}^{8,9}$ Comparado con los datos reportados en todo el mundo, en nuestro medio esta incidencia es menor (10.1\%). Esto concuerda con reportes latinoamericanos y nacionales ${ }^{8}$ en los que se ha remarcado mucho la menor presencia de inhibidor, lo cual se vuelve a comprobar en nuestros pacientes, sin encontrar una causa racial específica, sino solo una asociación al uso de factores de la coagulación derivados plasmáticos como principal tratamiento en profilaxis.

En cuanto a la profilaxis como estándar de tratamiento, el objetivo es que todos los pacientes se encuentren en profilaxis primaria para evitar o demorar la aparición de daño articular. Cuando se instauró este régimen de tratamiento se redujeron los sangrados articulares de un $30-40 \%$ a un $0-6 \%$ por año. ${ }^{10}$ En México, de acuerdo con las estadísticas de 2015 , solo el $15 \%$ de la población con hemofilia estaba en profilaxis, y de estos, la mayoría están siendo tratados en el IMSS. Se han hecho avances en cuanto a profilaxis que han permitido un incremento del consumo per cápita de factor VIII de 4 unidades, que es el ideal, aunque en la actualidad se barajan cifras de 2.1 unidades per cápita. ${ }^{8}$

\section{Conclusiones}

El método de evaluación por ultrasonido HEAD-US es una herramienta útil en la consulta de hematología pediátrica, adicional a las escalas clínicas que suelen ser subjetivas y cambiantes si hay sangrados agudos. La valoración por ultrasonido da un enfoque más objetivo al plasmar en imagen los cambios tempranos en la sinovial, el cartílago articular y el hueso subcondral. Al ser un estudio económico, asequible y disponible en la mayoría de los centros, debemos concientizar a nuestras autoridades de la importancia de implementarlo en la consulta del paciente con hemofilia. En nuestro servicio forma parte de la 
evaluación semestral del paciente, y aunque no tuvimos limitaciones con el equipo, es de recordar que el ultrasonido lleva una curva de aprendizaje. Los hematólogos estamos siendo entrenados por los radiólogos que a su vez se capacitaron en llevar este método, así que el principal problema al que nos enfrentamos inicialmente fue llevar a cabo las capacitaciones y que el radiólogo a su vez nos tomara bajo tutela y revisara nuestra forma de abordar al paciente. Esto implica al inicio un tiempo que de por sí en nuestras instituciones es reducido, pero a la larga rendirá frutos en los pacientes, ya que el personal capacitado estará adiestrado para realizar esta evaluación en aproximadamente 5 minutos.

Por otra parte, de acuerdo con nuestros resultados, el peso por arriba del percentil 50 es un factor de riesgo independiente de desarrollar complicaciones por sangrado articular, mientras que la edad y el tipo de hemofilia no parecen relacionados con la aparición de hemartrosis.

El ultrasonido articular realizado con método HEAD-US es una herramienta útil, eficaz, certera y accesible para la detección temprana de artropatía y hemartrosis, corroborando la enfermedad diagnosticada mediante exploración clínica e incluso cuando la clínica es negativa.

\section{Financiamiento}

La realización de este trabajo fue auspiciada por un donativo no restringido al grupo de trabajo por parte de Novo Nordisk México.

\section{Conflicto de intereses}

Los autores declaran no tener ningún conflicto de intereses.

\section{Responsabilidades éticas}

Protección de personas y animales. Los autores declaran que para esta investigación no se han realizado experimentos en seres humanos ni en animales.

Confidencialidad de los datos. Los autores declaran que han seguido los protocolos de su centro de trabajo sobre la publicación de datos de pacientes.

Derecho a la privacidad y consentimiento informado. Los autores han obtenido el consentimiento informado de los pacientes y/o sujetos referidos en el artículo. Este documento obra en poder del autor de correspondencia.

\section{Bibliografía}

1. Bakeer N. A new wave in the evaluation of haemophilic arthropathy. Haemophilia. 2017;23:491-3.

2. van Vulpen LFD, Holstein K, Martinoli C. Joint disease in haemophilia: pathophysiology, pain and imaging. Haemophilia. 2018;24 (Suppl 6):44-9.

3. Berges Garcia A, García Chávez J, Sánchez Jara B, Ortiz Torres MG, Garcidueñas Lozano M. Diagnóstico y Tratamiento de Hemofilia Hereditaria en < de 16 Años. Guía de Evidencias y Recomendaciones: Guía de Práctica Clínica. México, Instituto Mexicano del Seguro Social; 2018. Disponible en: http://imss.gob.mx/profesionales-salud/gpc.

4. Fischer $K$, Poonnoose $P$, Dunn AL, Babyn P, Manco-Johnson MJ, David JA, et al.; participants of the International Symposium on Outcome Measures in Hemophilic Arthropathy. Choosing outcome assessment tools in haemophilia care and research: a multidisciplinary perspective. Haemophilia. 2017:23:11-24.

5. Martinoli C, Della Casa Alberighi O, Di Minno G, Graziano E, Molinari AC, Pasta G, et al. Development and definition of a simplified scanning procedure and scoring method for Haemophilia Early Arthropathy Detection with Ultrasound (HEAD-US). Thromb Haemost. 2013;109:1170-9.

6. De la Corte-Rodríguez H, Rodríguez-Merchán EC, Álvarez-Román MT, Martín-Salces M, Martinoli C, Jiménez-Yuste V. The value of HEAD-US system in detecting subclinical abnormalities in joints of patients with hemophilia. Expert Rev Hematol. 2018;11:253-61.

7. De la Corte-Rodríguez H, Rodríguez-Merchán EC, Jiménez-Yuste V. Point-of-care ultrasonography in orthopedic management of hemophilia: multiple uses of an effective tool. HSS J. 2018;14:307-13.

8. Izquierdo Zarco M. Reporte sobre hemofilia en México. Federación de Hemofilia de la República Mexicana A. C. (Fecha de acceso 17-072018). Disponible en: http://docplayer.es/30186351-Reporte-sobre-hemofilia-en-mexico.html

9. Carcao M, Goudemand J. Inhibitors in hemophilia: a primer. Online treatment of hemophilia. (Fecha de acceso: 26-07-2018). Disponible en: http://www1.wfh.org/publication/files/pdf-1122.pdf

10. Abraham A, Apte S, Sunder A, Subramaniam A, Korula A, Joshi A, et al. Meaningful reduction of annual bleeding rate with lower dose prophylaxis in minimally treated children with hemophilia in India. Blood. 2017;130 (Suppl 1):1079. 